\title{
Erratum
}

\section{On shear-induced turbulence in rotating stars}

\author{
S. Mathis ${ }^{1,2,3}$, A. Palacios ${ }^{3}$, and J.-P. Zahn ${ }^{2}$ \\ 1 Observatoire de Genève, 51 chemin des Maillettes, 1290 Sauverny, Switzerland \\ 2 LUTH, Observatoire de Paris, 92195 Meudon, France \\ 3 CEA/DSM/DAPNIA/Service d'Astrophysique, L'Orme des Merisiers, Bât. 709, 91191 Gif-sur-Yvette, France \\ e-mail: ana.palacios@cea.fr
}

(A\&A, 425, 243-247 (2004), DOI: 10.1051/004-6361:20040279)

Key words. turbulence - stars: evolution - errata, addenda

We discovered an error in the coding of the expression of the effective diffusivity $D_{\text {eff }}$, which lead to wrong conclusions about the impact of the enhanced horizontal diffusivity $D_{\mathrm{h}}$ on the overall vertical mixing. Contrary to what we concluded in the original paper (A\&A 425, 243, 2004), the mixing due to meridional circulation is reduced by the use of the new prescription (Eq. (19)).

The three figures of the original paper have been corrected accordingly and are given here at slightly different ages.

According to our corrected computations, the last sentence of the Abstract should be replaced by: "its implementation in a stellar evolution code leads to reduced mixing as illustrated by models of a rotating main sequence star of 1.5 solar masses."

On page 246, Col. 2, at the second paragraph.

"The second effect is to reduce the latitudinal fluctuations of chemical composition, which otherwise would tend to suppress the meridional circulation, as was pointed out already by Mestel (1953). The consequence is an increase of the circulation speed $U_{2}$, which compensates that of $D_{\mathrm{h}}$, and this explains why the effective diffusivity $D_{\text {eff }}$ is less affected than one would expect by the increase of $D_{\mathrm{h}}$, as can be seen in Fig. 2."

should be replaced by

"As expected from Eq. (14), the high values obtained for the horizontal component of the turbulent diffusivity lead to low values of the effective diffusivity. As shown in Fig. 2, $D_{\text {eff }}$ becomes negligible compared to $D_{\mathrm{v}}$ when adopting the new prescriptions for $v_{\mathrm{h}}$, whether from Maeder (2003) or the one given in Eq. (19)."

Finally, in the Conclusions, the last sentence of the first paragraph "We have analysed the effects of this dramatic change in $D_{\mathrm{h}}$ on the structure of a $1.5 M_{\odot}$ star: it increases the vertical transport of matter, and therefore enhances the mixing,

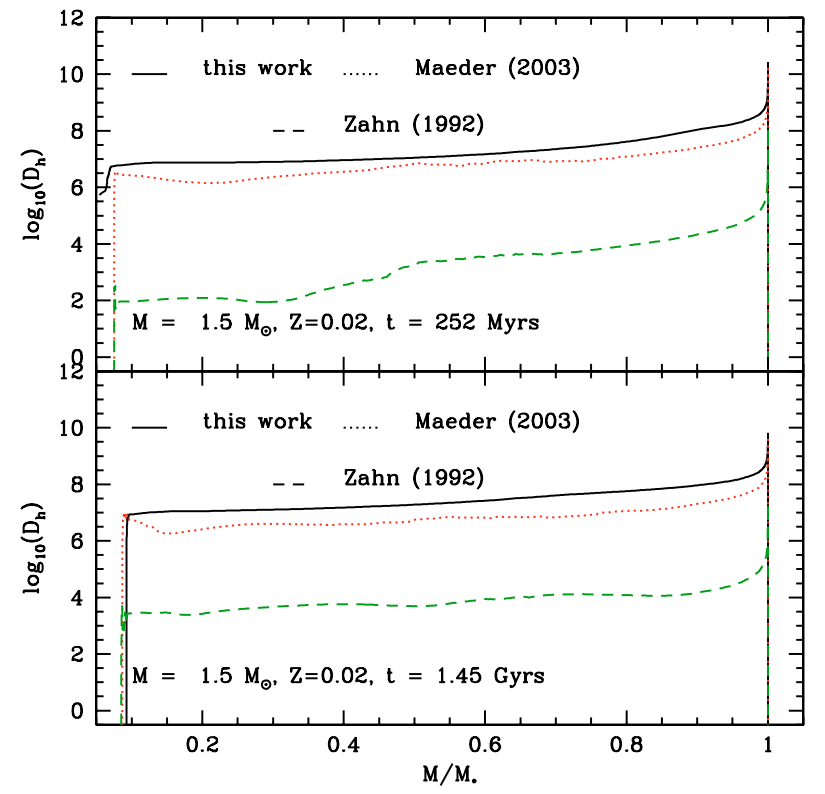

Fig. 1. Horizontal component $D_{\mathrm{h}}$ of the turbulent diffusivity generated by the horizontal shear in a $1.5 M_{\odot}$ star rotating initially with an equatorial velocity of $110 \mathrm{~km} \mathrm{~s}^{-1}$, at two evolutionary stages $(0.252$ and 1.45 Gyr). Comparison of the old prescription (Eq. (13); Zahn 1992) with that of Eq. (15) (Maeder 2003), and that of the present paper.

particularly as the star evolves and a molecular weight gradient is building up."

should be replaced by

"We have analyzed the effects of this dramatic change in $D_{\mathrm{h}}$ on the structure of a $1.5 M_{\odot}$ star, and find that it decreases the meridional circulation contribution to the vertical transport of matter. Contrary to what is obtained with the old prescription 


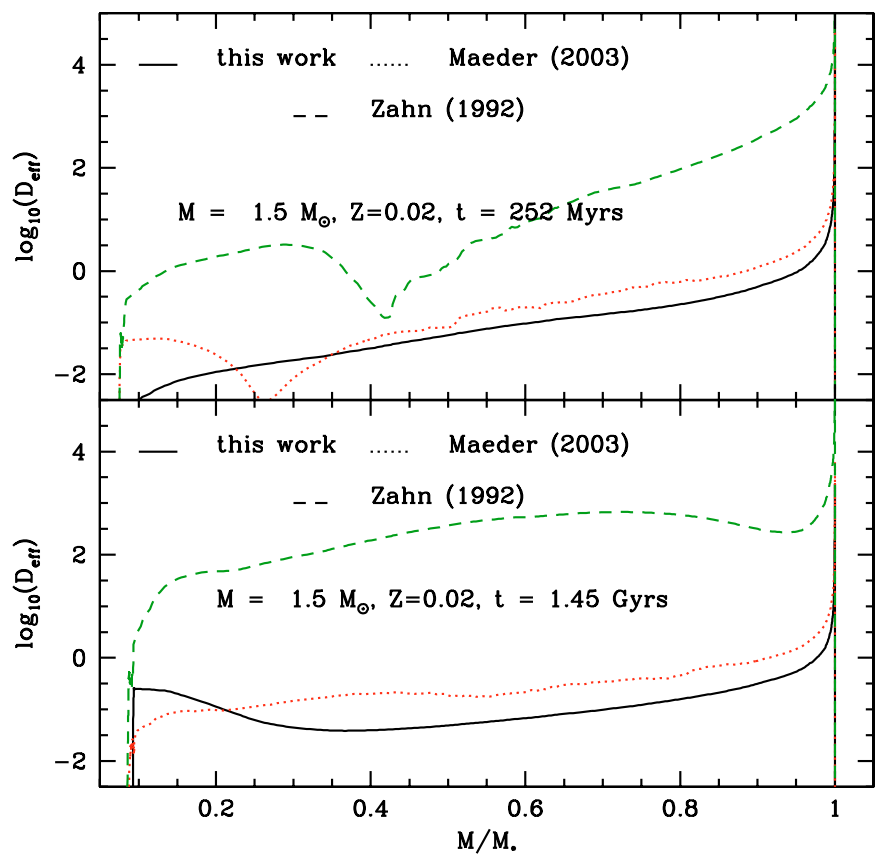

Fig. 2. Same comparison as Fig. 1 for the effective diffusivity $D_{\text {eff }}$ in the vertical direction, which results from the erosion of the advective transport through the horizontal diffusivity $D_{\mathrm{h}}$ displayed in Fig. 1. Here, $D_{\text {eff }}$ has been evaluated using the old prescription for $D_{\mathrm{h}}$ (Zahn 1992), that of Maeder (2003), and that of the present paper (Eq. (19)).

for $D_{\mathrm{h}}$, the effective coefficient remains small compared to the vertical turbulent coefficient during the evolution on

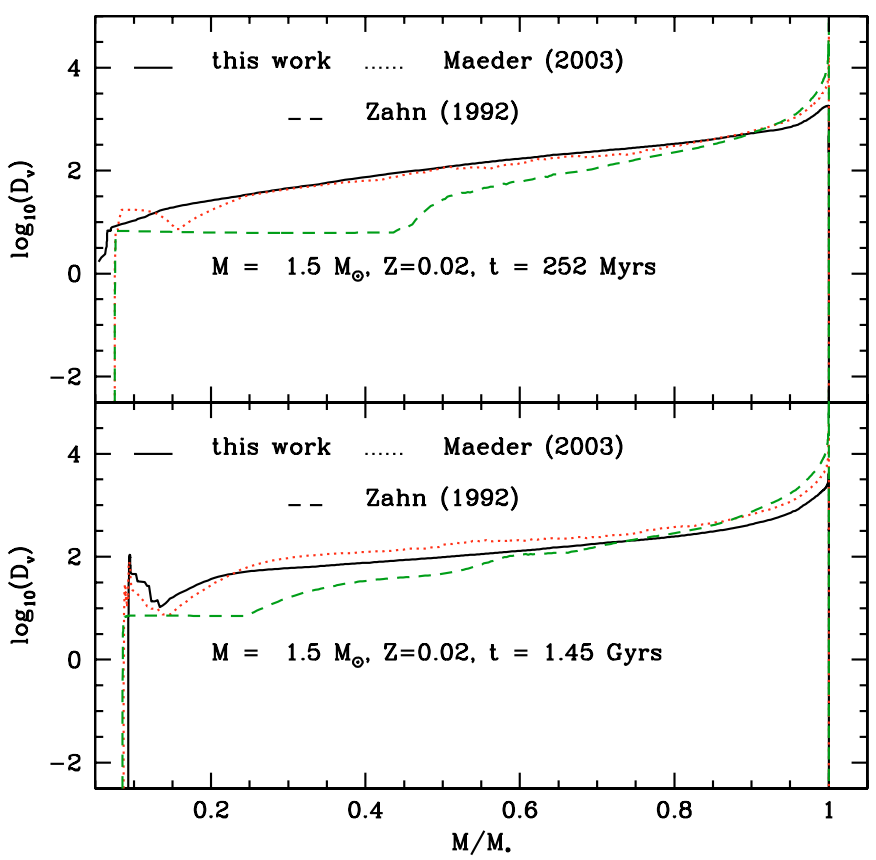

Fig. 3. Same comparison as Figs. 1 and 2 for the vertical component $D_{\mathrm{v}}$ generated by the vertical shear. $D_{\mathrm{v}}$ depends on $D_{\mathrm{h}}$ when there is a vertical composition gradient, according to Eq. (7).

the main sequence, even when a molecular weight gradient is building up. Thus, the overall mixing is reduced when using the new $D_{\mathrm{h}}$ values." 